\title{
Conductas alimentarias de riesgo y su relación con la imagen corporal en estudiantes de enfermería
}

\author{
María del Pilar Ureña-Molina* \\ Mayerlin Pacheco-Milian** \\ Mónica Janett Rondón-Ortega ${ }^{* * *}$
}

Recibido:

30 de Septiembre

de 2015

\section{RESUMEN}

Objetivo: determinar la relación entre las conductas alimentarias de riesgo y la imagen corporal en los estudiantes de enfermería durante el primer semestre del año 2015. Materiales y Métodos: estudio de tipo cuantitativo, correlacional. Se utilizaron como instrumentos, el Test de Imagen Corporal - Cuestionario Body Shape Questionnaire y el Test De Actitudes Alimentarias - 26. La muestra estuvo conformada por 181 estudiantes. Resultados: la mayor proporción de estudiantes pertenecen al género femenino, las edades fluctuaron entre los 16 a 28 años, predominando el estado civil soltero, pertenecientes a un estrato socioeconómico bajo (1 -2) y conviven con sus padres. El $63 \%$ obtuvo un estado nutricional normal, la mayor parte de ellos no presentaron conductas alimentarias de Riesgo (94\%) y se encontraban satisfechos con su imagen corporal (91\%). Se estableció una relación estadísticamente significativa entre las conductas alimentarias de riesgo y la imagen corporal. Conclusiones: los estudiantes de enfermería, en su mayoría, no presentaron conductas alimentarias de riesgo, es decir, no realizan prácticas extremas para el control de su peso corporal y los que la presentaron, pueden tener afectaciones en su desempeño académico, las relaciones interpersonales y la calidad de vida. Así mismo, la mayoría de ellos mostraron una apreciación satisfactoria de su imagen corporal y un pequeño porcentaje, estuvo insatisfecho, reflejando diversos desórdenes de tipo conductual, cognitivo y emocional. Se determinó que la integridad de la imagen corporal disminuye la presencia de conductas alimentarias de riesgo.

PALABRAS CLAVE: actitud frente a la salud, enfermería, hábitos alimenticios, satisfacción personal ${ }^{* * * *}$. 



\section{Comportamentos alimentares de risco e sua relação com a imagem corporal entre estudantes de enfermagem com}

ISSN 1794-9831

E-ISSN 2322-7028

Vol. 12 No. 2

Jul - Dic 2015

Cúcuta, Colombia

\section{RESUMO}

Objetivo: para determinar a relação entre transtornos alimentares e imagem corporal em estudantes de enfermagem durante o primeiro semestre de 2015. Materiais e Métodos: quantitativa, de correlação. Eles foram usados como instrumentos de teste de imagem corporal - Body Shape Questionnaire Questionnarire e Eating Attitudes Test - 26. A amostra foi composta por 181 alunos. Resultados: a maior proporção de estudantes são do sexo feminino, a idade variou entre 16-28 anos, estado civil, principalmente único, pertencente a um baixo nível socioeconômico ( 1 -2) e viveu com seus pais. $63 \%$ tiveram um estado nutricional normal, a maioria de les não apresentava risco comportamentos alimentares (94\%) e foram satisfeitos com a sua imagem corporal (91\%). Uma relação estatisticamente significativa entre a imagem corporal transtornos alimentares e é estabelecido. Conclusões: o estudantes de enfermagem em sua maior parte apresentou nenhum risco comportamentos alimentares, o que significa que não realizam práticas extremas para controlar seu peso corporal e os que apresentavam pode ter danos em seu desempenho acadêmico, relações interpessoais e qualidade de vida. Da mesma forma, a maioria deles mostrou uma avaliação satisfatória da imagem corporal e uma pequena percentagem, estava insatisfeito, refletindo vários distúrbios comportamentais, cognitivos e emocionais. Determinou-se que a integridade da imagem corporal diminui a presença de distúrbios alimentares.

PALAVRAS-CHAVE: atitude para a saúde, nutrição, hábitos alimentares, a satisfação pessoal. 
ISSN 1794-9831

E-ISSN 2322-7028

Vol. 12 No. 2

Jul - Dic 2015

Cúcuta, Colombia

\section{INTRODUCCIÓN}

L

os jóvenes universitarios estudiantes de enfermería, usualmente al encontrarse en esta etapa de la vida y al pertenecer a una carrera universitaria que presenta una marcada preferencia por el género femenino, manifiestan algunos patrones particulares de comportamiento que buscan impactar o ser aceptados en el círculo social que los rodea, mediante el mantenimiento de una imagen corporal delgada y tonificada, considerada como el modelo más cercano a la perfección de la belleza física de las personas y convirtiéndose en la principal motivación para elegir ciertas conductas alimentarias no saludables.

Un fenómeno similar acontece, paradójicamente, cuando muchos de estos jóvenes pierden el interés por su imagen corporal y también desarrollan hábitos alimenticios inadecuados. Lo anterior genera, en la mayoría de los estudiantes, la posibilidad de desarrollar conductas alimentarias de riesgo que conllevan a una alteración en su salud física y mental de una manera preocupante. Diagnosticar tempranamente las conductas alimentarias de riesgo permite brindar a tiempo un adecuado tratamiento y, a su vez, instaurar medidas preventivas en la universidad -donde estos jóvenes permanecen gran parte de su tiempo-, con estrategias que promuevan hábitos saludables y el cuidado de sí mismos, de modo que se favorezca un entorno universitario sano.

La conducta se define como la modalidad que tiene una persona para comportarse en diversos ámbitos de su vida, determinada por los factores genéticos, psicológicos, económicos e intereses culturales $\mathrm{y}$ sociales de cada individuo (1). Dentro de las conductas que puede asumir un individuo están las alimentarias, que pueden ser definidas como un comportamiento habitual relacionado con hábitos alimentarios, selección de alimentos, preparaciones y volúmenes consumidos, que influye directamente en el estado nutricional de los individuos pertenecientes a diversos grupos etarios (2).

La alteración de estas conductas ha dado origen a lo que se conoce como conductas alimentarias de riesgo (CAR), entre las cuales se encuentran los atracones o episodios de ingestión excesiva, la sensación de pérdida de control al comer, el seguimiento de dietas restringidas, los ayunos, el vómito auto-inducido, el abuso de laxantes, diuréticos y anfetaminas, y el ejercicio físico en exceso, conductas realizadas con la finalidad de perder peso o mejorar la figura corporal (3). Desde esta perspectiva, las personas que presentan este tipo de conductas padecen. en su mayoría. de algún trastorno alimenticio, ya que las CAR son características o manifestaciones propias de éstos últimos.

Los trastornos de la conducta alimentaria (TCA) se han convertido en un serio problema para la salud pública en todas la naciones del mundo. Están representados, en su mayoría, por la anorexia nerviosa $(\mathrm{AN})$ y la bulimia nerviosa $(\mathrm{BN})$, así como por las manifestaciones intermedias o trastornos de la conducta alimentaria no especificados (TCANOES), considerados como enfermedades crónicas y progresivas en las que prevalece una alteración o distorsión de la autoimagen corporal, causada por factores de riesgo biológicos (alteraciones genéticas y neurológicas, género y etapa de la vida), psicológicos y sociales (sensación de fracaso, estrés, ansiedad, depresión, abuso físico o sexual, problemas familiares, comparación social, burlas de pares y familiares, baja autoestima e insatisfacción corporal) (4).

La AN se define como un estado de inanición voluntaria que produce una pérdida significativa de peso y se cree que es inducida por una distorsión que se tiene de la propia imagen. La BN se caracteriza por dietas restrictivas que no llevan al individuo al peso deseado, situación frustrante que lo conduce a consumir grandes cantidades de comida en un lapso de tiempo corto, seguida por una intensa culpabilidad que lo lleva a utilizar mecanismos como laxantes, purgas y vómitos para eliminar el exceso de alimentos. Finalmente, los TCA NOES incluyen pacientes que no cumplen todos los criterios para ser diagnosticados como AN o BN (5).

Desde el ámbito mundial se puede mencionar que en Estados Unidos, para el año 2011, alrededor de siete millones de mujeres y un millón de varones padecieron algún TCA. Ese año, entre un $10 \%$ y un $25 \%$ de norteamericanos que padecieron de anorexia murieron a causa de esta enfermedad (6). En cuanto a la prevalencia de $\mathrm{BN}$ en estudiantes universitarias americanas fue de un $19 \%$ y de anorexia entre un $1 \%$ al $4.1 \%$ (7). Así mismo, los estudios de prevalencia clínica de trastornos de la alimentación indican que 
entre $5 \%$ y un $10 \%$ de todos los individuos que padecen estos trastornos, son hombres (8).

En el escenario Europeo se ha estimado, para el 2009, que entre el $0.3 \%$ y el $2.2 \%$ de mujeres jóvenes sufren de AN y del $1 \%$ al $2 \%$ de BN. La mortalidad de anorexia es muy alta y equivale al $5 \%$ (9). En el ámbito latinoamericano, en Argentina, entre el $12 \%$ y el $15 \%$ de los adolescentes padecen de anorexia o bulimia nerviosa, siendo el $90 \%$ de los afectados mujeres y el $10 \%$ varones (10). De igual manera, la cantidad de varones con estos trastornos ha ido en aumento.

En Colombia se estima que las cifras de prevalencia de los trastornos de la alimentación, según las investigaciones del Departamento de Psiquiatría de la Universidad de Antioquia, llegarían a una tasa del 38 $\%$ y, a su vez, que los porcentajes de anorexia serían iguales a los que se manejan a nivel mundial, del $1 \%$ al $4 \%(11)$.

Es importante, por tanto, detectar tempranamente las conductas alimentarias de riesgo, permitiendo ofrecer un adecuado tratamiento a tiempo y, a su vez, que se instauren medidas preventivas, con estrategias que promuevan hábitos saludables y el cuidado de sí mismo, de modo que se favorezca un entorno más sano y favorable para los jóvenes universitarios, en especial los estudiantes de enfermería.

\section{MATERIALES Y MÉTODOS}

Se utilizó una metodología cuantitativa de tipo correlacional. Los diseños de investigación cuantitativos generalmente reflejan una filosofía determinista, la cual está basada en el paradigma del pensamiento pos-positivista, el cual examina la causa y la manera en que diferentes acciones o fenómenos interactúan y/o influyen en los resultados. El enfoque es típicamente deductivo, donde la mayor parte de las ideas o conceptos se reducen a variables y las relaciones entre ellas son sometidas a test. El conocimiento resultante es basado en la observación, medición e interpretación cuidadosa de la realidad objetiva (12).

Los estudios correlaciónales involucran la investigación sistémica de la naturaleza de relaciones o asociaciones entre las variables. Los diseños de correlación son típicamente transversales y son utilizados para examinar si los cambios en una o más variables están relacionados a los cambios en otra(s) variable(s) (12).

La población estuvo compuesta por 330 estudiantes pertenecientes al programa de enfermería de una universidad, matriculados en el primer semestre del año 2015. La muestra estuvo integrada por 181 estudiantes, escogidos por muestreo probabilístico estratificado, seleccionando la muestra representativa por cada semestre y teniendo en cuenta que fueran estudiantes del programa de enfermería y accedieran a participar voluntariamente. A continuación, las fórmulas utilizadas fueron: en este estudio fueron:

$$
\begin{aligned}
& \underline{n=N+Z 2 . p .(1-p) E 2(N-1)+Z 2 . p .(1-P)} \\
& n=330(0,05) 2(330-1)+1 \\
& n=330(0,0025)(329)+1 \\
& n=330(0,8225)+1 \\
& n=3301,8225 \\
& n=181
\end{aligned}
$$

$\mathrm{N}^{\circ}$ de Estudiantes en cada Estrato (Semestre) $=\mathrm{N}^{\circ}$ Total de estudiantes en cada semestre / Total de la Población* $\mathrm{N}^{\circ}$ de la Muestra

Primer Semestre: $42 / 330 * 181=23$

Segundo Semestre: $39 / 330 * 181=21$

Tercero Semestre: $36 / 330 * 181=20$

Cuarto Semestre: $40 / 330 * 181=22$

Quinto Semestre: $45 / 330 * 181=25$

Sexto Semestre: $46 / 330 * 181=25$

Séptimo Semestre: $31 / 330 * 181=17$

Octavo Semestre: $51 / 330 * 181=28$

La recolección de los datos se realizó por medio del Test de Imagen Corporal - Cuestionario B.S.Q. (BodyShapeQuestionnarire) y el Test de Actitudes Alimentarias EAT-26. El Test de Imagen Corporal es un cuestionario auto-administrado con un Alpha de Cronbach de 0.95, compuesto por 34 ítems con opción de respuesta tipo Likert de 6 puntos, que va
ISSN 1794-9831

E-ISSN 2322-7028

Vol. 12 No. 2

Jul - Dic 2015

Cúcuta, Colombia 
ISSN 1794-9831

E-ISSN 2322-7028

Vol. 12 No. 2

Jul - Dic 2015

Cúcuta, Colombia desde Nunca hasta Siempre; al finalizar la sumatoria total de los puntos del test, un puntaje por encima de 105 puntos se considera como insatisfacción de la imagen corporal. El objetivo de este test consiste en determinar si existe o no insatisfacción con la imagen corporal (13). Este instrumento ha sido utilizado en países como Cuba (14), México (15) y Colombia (16-17).

El Test de Actitudes Alimentarias EAT-26 ha sido considerado como una de las pruebas estandarizadas más empleadas para estudiar las conductas alimentarias de riesgo. Evalúa tres factores en los cuales se evidencian conductas alimentarias de riesgo: Factor $\mathrm{N}^{\circ} 1$, la dieta relacionada con la evitación de alimentos que engordan y la preocupación por estar delgado(a); Factor $\mathrm{N}^{\circ} 2$, Bulimia y preocupación por la comida, donde se reflejan pensamientos sobre la comida e indicadores de bulimia y Factor $\mathrm{N}^{\circ} 3$, control oral, que está relacionado con el autocontrol en el comer (18-19).

Este test fue elaborado por David Garner y Paul Garfinkel en 1979. En 1982 se construyó la segunda versión que consta de 26 ítems que indican comportamientos, los cuales cuentan con una correlación de 0.98 respecto al EAT-40 (primera versión). Se contesta mediante una escala tipo Likert de 6 categorías. El puntaje final de la prueba corresponde a la suma de todos los ítems, cuyo total puede variar entre 0 y 78 puntos. El obtener un puntaje igual o superior a 20 puntos se asocia con conductas alimentarias de riesgo (18). El instrumento cuenta con un nivel de confiabilidad mediante el Alpha de Cronbach de 0.74 (19).

El test ha sido utilizado como una herramienta de screening para determinar el Riesgo de Desorden Alimentario en colegios, universidades y otros grupos especiales de riesgo como los atletas. Al ser una prueba de tamizaje (screening), se asume que una anticipada identificación de un desorden alimentario puede conducir a un tratamiento temprano, reduciendo así ciertas complicaciones físicas y psicológicas e incluso la probabilidad de muerte. Este instrumento ha sido utilizado en países como Chile (18), Brasil (19), Paraguay (20), España (10), y Colombia (1521).

Para la aplicación de los instrumentos, el Test de
Imagen Corporal - Cuestionario B.S.Q contó con el permiso de aplicación pormedio del correo electrónico, donde sus autores aceptaron la respectiva solicitud, mientras que el Test de Actitudes Alimentarias EAT26 se encuentra gratuitamente disponible al público en general $\mathrm{y}$, por consiguiente, no se presentan repercusiones legales para su uso. El desarrollo del proyecto contó con el aval del comité curricular del programa de enfermería de la universidad objeto de estudio.

Los resultados obtenidos se analizaron sistemáticamente mediante el programa SPSS, versión 22 para Windows en español, representando la información recolectada en tablas y gráficas. Para el cruce de las variables conductas alimentarias de riesgo e imagen corporal se utilizó la prueba estadística de Chi Cuadrado (X2 > 3.84), que determina cuál de las hipótesis planteadas es la correcta, es decir, si existe o no existe relación estadísticamente significativa entre las dos variables.

El estudio se realizó teniendo en cuenta las normas éticas para la investigación con seres humanos, garantizando la total privacidad y no divulgación de los resultados con nombre propio. Además, se tuvieron en cuenta valores éticos como la autonomía, no maleficencia, beneficencia, justicia y confidencialidad; el estudiante obtuvo pleno conocimiento de los objetivos del estudio y riesgos, firmando el consentimiento informado (22).

\section{OBJETIVOS}

\section{Objetivo general}

Determinar la relación entre las conductas alimentarias de riesgo y la imagen corporal de los estudiantes de enfermería de una universidad en el primer semestre del año 2015.

\section{Objetivos específicos}

- Caracterizar socio demográficamente a los estudiantes de enfermería.

- Clasificar el estado nutricional de los estudiantes de enfermería.

- Identificar las conductas alimentarias de riesgo de los estudiantes de enfermería. 
- Identificar la imagen corporal de los estudiantes de enfermería.

\section{RESULTADOS}

\section{Caracterización sociodemográfica de la población estudiantil}

En el estudio participaron 181 estudiantes de una universidad pública, cursando los semestres de I a VIII del programa de enfermería, donde el género que predominó fue el femenino, con un $81 \%$. La edad promedio fue de 19 años, en un rango de 16 a 28 años. El $58 \%$ de los estudiantes son adultos jóvenes y el $48 \%$ adolescentes. Respecto al nivel económico predominante fue el nivel Bajo (1-2), con un $73 \%$. El estar en condición de soltero fue el estado civil que más prevaleció con un $94 \%$ del total de la población muestral y el $71,8 \%$ de los estudiantes residían con sus padres en el área urbana.

\section{Clasificación del estado nutricional de los estudiantes}

Con relación al índice de Masa Corporal (IMC), en este estudio se evidenció que la mayoría de la población estudiada (64\%) presentaba un estado nutricional normal, el $21 \%$ sobrepeso, el $14 \%$ bajo peso y, finalmente, el $1 \%$ se encontraba en obesidad tipo 1 , como lo referencia la figura 1 .

Figura 1. Caracterización por índice de masa corporal (IMC) de los estudiantes de enfermería en el primer semestre del 2015.

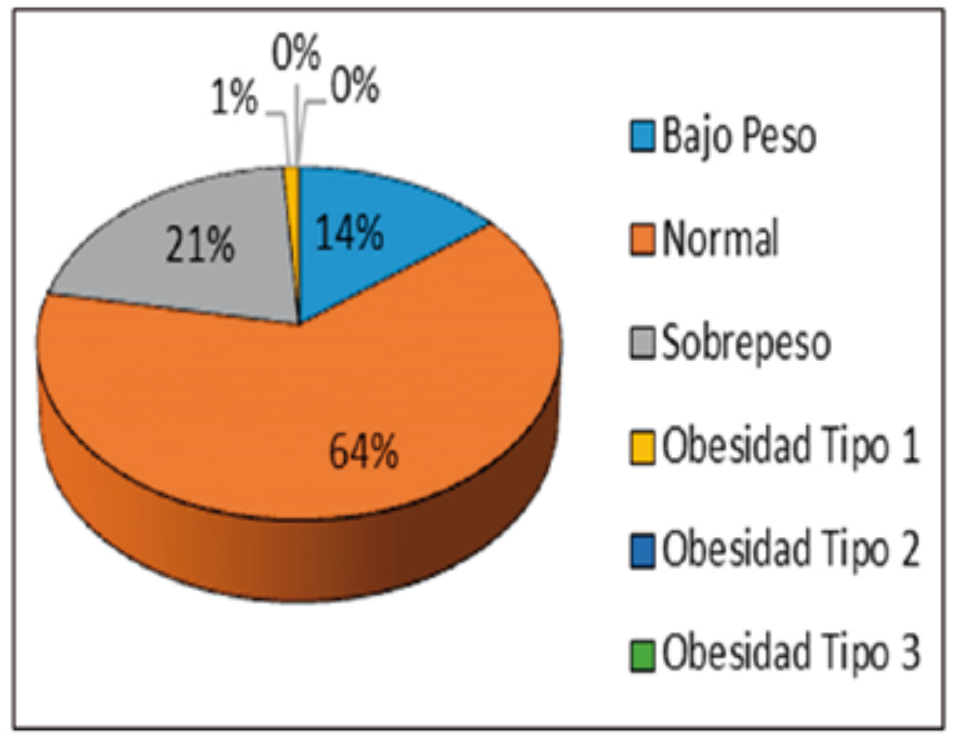

Fuente: Adaptación del Test de Actitudes Alimentarias (EAT-40), 1991.

Identificación de las conductas alimentarias de riesgo en los estudiantes

En cuanto a las conductas alimentarias de riesgo se pudo evidenciar que el $94 \%$ de los participantes presentaron conductas alimentarias sin riesgo, mientras que el restante $6 \%$ sí las manifestó, como se observa en la figura 2. Es decir, la mayor proporción de estudiantes cuentan con comportamientos alimentarios favorables, evitando el riesgo de recaer en un trastorno de la conducta relacionado con la alimentación; y una pequeña pero alarmante parte de ellos, en alguna medida, se encuentra en riesgo al presentar preocupaciones por engordar, episodios de atracones con sensación de falta de control al comer, dietas restrictivas, ejercicio físico excesivo, ingesta de medicamentos o consumo de pastillas para adelgazar.
ISSN 1794-9831

E-ISSN 2322-7028

Vol. 12 No. 2

Jul - Dic 2015

Cúcuta, Colombia 
Figura 2. Conductas alimentarias de los estudiantes de enfermería del primer semestre del año 2015.

Vol. 12 No. 2

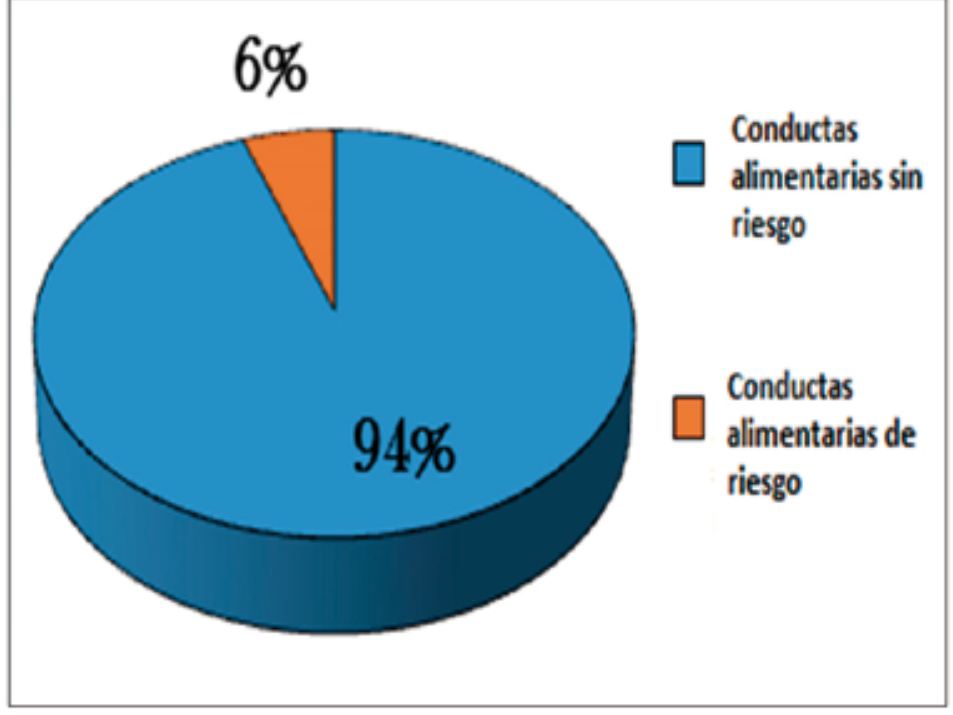

Fuente: Adaptación del Test de Actitudes Alimentarias (EAT-40), 1991.

Identificación de la imagen corporal en los estudiantes

Con respecto a la imagen corporal, se puede evidenciar que el $91 \%$ de los participantes mostró satisfacción con su imagen corporal, es decir, evidenció aceptación, placer y gusto por la expresión de su cuerpo, mientras que el $9 \%$ manifestó lo contrario (figura 3). Teniendo en cuenta que esta apreciación depende de los componentes perceptivos - cognitivos y afectivos que cada persona tiene de sí misma, las sobreestimaciones o subestimaciones de su propio cuerpo pueden aumentar el riesgo de los estudiantes a padecer problemas psicosociales

Figura 3. Satisfacción de la imagen corporal de los estudiantes de enfermería del primer semestre del año 2015.

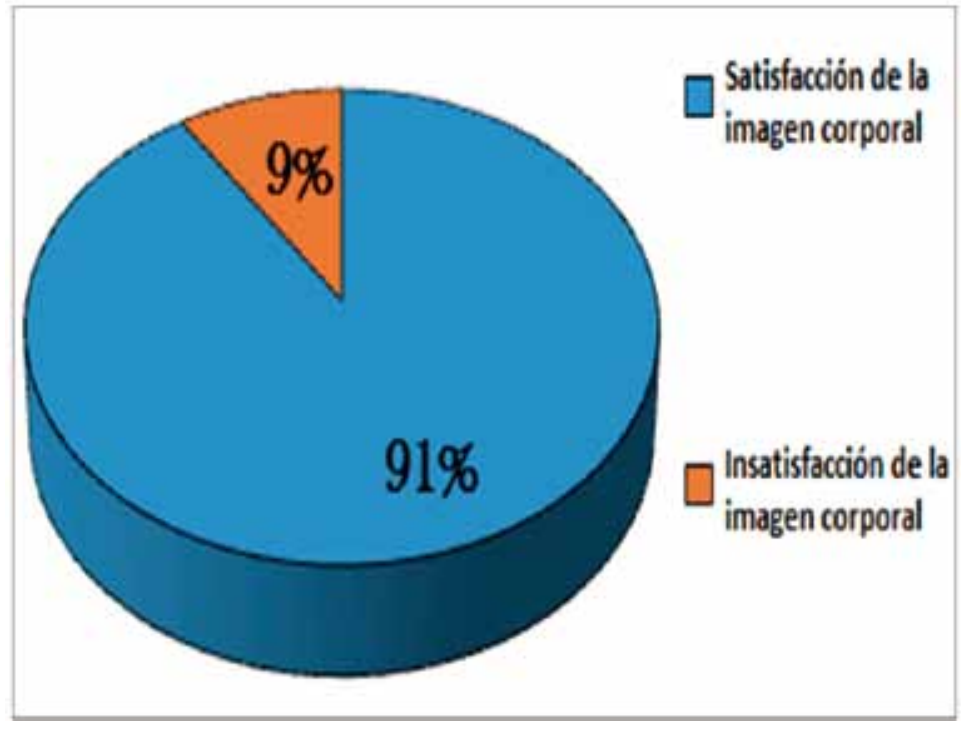

Fuente: Test de Imagen Corporal (BSQ), 1987. 


\section{Relación entre conductas alimentarias de riesgo con la imagen corporal}

En la figura 4 se puede observar que a mayor presencia de conductas alimentarias sin riesgo mayor es la satisfacción de la imagen corporal, en un $89 \%$ de los estudiantes, $\mathrm{y}$, de manera contraria, el presentar conductas alimentarias riesgosas aumenta la insatisfacción con la imagen corporal en un $4 \%$.

Al establecer la relación entre las conductas alimentarias de riesgo y la imagen corporal se encontró un resultado de Chi Cuadrado de X2 $=45,688$ (existe relación $>3,84$ ), es decir, existe una relación estadísticamente significativa entre las conductas alimentarias de riesgo y la imagen corporal.

Figura 4. Relación entre conductas alimentarias e imagen corporal de los estudiantes de enfermería del primer semestre del año 2015.

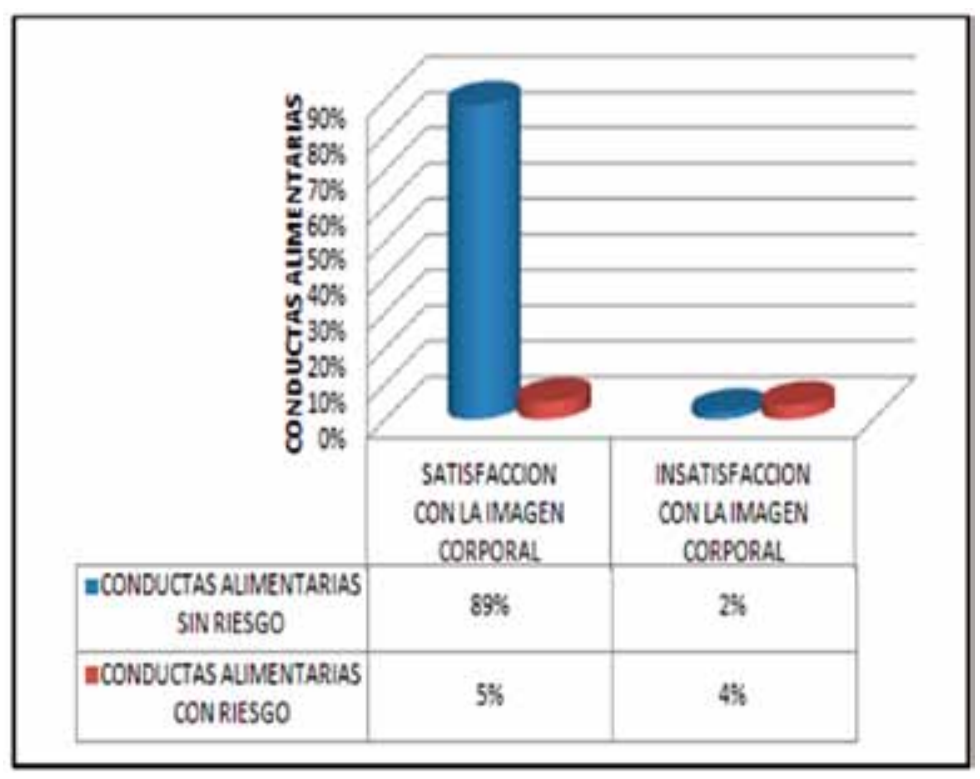

Fuente: Adaptación del Test De Actitudes Alimentarias (EAT-40), 1991; Test de Imagen Corporal (BSQ), 1987.

\section{DISCUSIÓN}

La aparición de conductas alimentarias de riesgo y la preocupación por la imagen corporal pueden variar según el sexo, debido al interés y características que cada joven tiene de acuerdo con su etapa de desarrollo. Hombres y mujeres se ven influenciados por los medios de comunicación y el deseo de buscar cuerpos perfectos: en las mujeres prevalecen las ideas de belleza relacionadas con la delgadez y es por eso que llevan a cabo conductas orientadas al control de su peso, mientras que en los hombres es menos frecuente la existencia de esta problemática y la tendencia se ve orientada a obtener un cuerpo musculoso y voluminoso (22).

La población universitaria está constituida, en su gran mayoría, por adolescentes y adultos jóvenes, edades que son consideradas de alto riesgo para adquirir conductas alimentarias inadecuadas debido a los cambios que suceden a nivel físico y hormonal en esta etapa del ciclo vital.

Durán et al. (23) expresan que la población adolescente y joven, con frecuencia, está insatisfecha con su imagen corporal, fenómeno que los hace más vulnerables ante cualquier método de adelgazamiento. De igual manera, el entorno universitario, en cierta medida, es generador de hábitos de vida que pueden influir en la adopción de comportamientos inapropiados para el cuidado de su propia salud. Según la investigación de Bernández et al. (24), el ambiente universitario propicia situaciones estresantes que pueden alterar el funcionamiento psicológico y somático
ISSN 1794-9831

E-ISSN 2322-7028

Vol. 12 No. 2

Jul - Dic 2015

Cúcuta, Colombia 
ISSN 1794-9831

E-ISSN 2322-7028

Vol. 12 No. 2

Jul - Dic 2015

Cúcuta, Colombia de los estudiantes, encontrándose que la mayor insatisfacción corporal se encuentra en mujeres de 18 y 20 años de edad.

El nivel social de los estudiantes de las universidades públicas es, sin duda, un aspecto importante en todos los estudios que se realizan. Interesa establecer el tipo de estudiantes que, desde el punto de vista de la capacidad económica de sus familias, ingresa a estas instituciones, lo que puede influir en el modo de alimentación o estilo de vida que lleva cada uno (25).

El nivel socioeconómico medio y alto guarda estrecha relación con mayores probabilidades de llevar a cabo conductas alimentarias de riesgo además de dificultades con la imagen corporal. No obstante, esta situación ha variado con el tiempo, presentándose en todas las clases sociales, sin distinción alguna. Se ha establecido en otros estudios $(15,26)$ que existe una asociación significativa entre conductas alimentarias de riesgo o trastornos de la conducta alimentaria y los estratos altos, así como también entre el impulso a la delgadez y el riesgo de conductas alimentarias de riesgo, puesto que ambas se incrementan a medida que el nivel social aumenta $(27,15,16,28)$.

En cuanto al índice de masa corporal (IMC) encontrado en los estudiantes de enfermería analizados, predominó un estado nutricional normal (64\%), encontrándose similitud con otros estudios $(29,30,5)$. Estos hallazgos demuestran que, con el paso de los años, la concientización por lograr una mejor calidad de vida ha influenciado la adquisición de prácticas más saludables como tener buenos hábitos alimenticios, realizar ejercicio frecuentemente e ir a chequeos médicos matutinos, entre otras, que generan en el individuo una satisfacción sobre su imagen corporal y, a su vez, una reducción en la incidencia de conductas alimentarias de riesgo y trastornos de la conducta alimentaria.

Las conductas alimentarias de riesgo fueron identificadas en un porcentaje muy pequeño de estudiantes $(6 \%)$, predominando las conductas $\sin$ riesgo. Resultados similares fueron hallados en el estudio realizado por Sáenz et al. (21), donde el 92,2 $\%$ de la población presentó conductas alimentarias sin riesgo, aunque discrepando un poco con los hallazgos de Moreno y Ortiz (16) y Hernández y Londoño (14) donde el $84 \%$ y el $87.3 \%$, respectivamente, presentaron conductas alimentarias sin riesgo.
El estudio realizado por Cano et al. (27) mostró cifras porcentuales más altas con respecto a las anteriores investigaciones, donde la frecuencia de las conductas alimentarias de riesgo fue de un $63 \%$.

En cuanto al tipo de conducta de riesgo, el estudio realizado por Franco et al. (28) refiere que las principales conductas de riesgo que efectúan los estudiantes son las dietas restrictivas, el atracón, las conductas compensatorias (vómito auto-inducido, ayuno, consumo de laxantes y/o diuréticos), las prácticas de ejercicios con la finalidad de quemar calorías y, finalmente, el consumo de productos para adelgazar. Estas conductas son asociadas a obesidad, sobrepeso y bajo peso.

De acuerdo con los resultados, son notorias las conductas alimentarias positivas en los estudiantes de enfermería del estudio, pudiéndose inferir que el pertenecer al área de la salud -específicamente a enfermería, donde los conceptos sobre el cuidado se tratan cotidianamente de forma integral y holística, y el abordaje de la promoción y prevención de las enfermedades debe realizarse en todas las etapas del ciclo vital y en todos los procesos de desarrollo- ha permitido una reducción de trastornos de la conducta alimenticia y, a futuro, de las enfermedades crónicas.

No obstante, es importante advertir que el $6 \%$ de los estudiantes presentaron conductas alimentarias de riesgo, las cuales representan un peligro para su salud, puesto que se encuentran expuestos a desarrollar trastornos del comportamiento alimentario (TCA) tales como anorexia nerviosa, bulimia nerviosa o trastorno por atracón.

Al igual que las conductas alimentarias sin riesgo, la imagen corporal fue percibida satisfactoriamente por el mayor número de estudiantes. Al comparar estos resultados, se pudieron evidenciar hallazgos similares con lo reportado por Trejo et al. (29) y Franco et al. (28), en cuyos estudios la satisfacción con la imagen corporal fue de $87.7 \%$ y $82.3 \%$ respectivamente. Sin embargo, difieren con estudios donde se mostraron cifras más elevadas con respecto a la presencia de la insatisfacción con la imagen corporal en los estudiantes, como es el caso de las investigaciones de Caballero (31) y Soto et al. (32) donde se encontró que el $34.6 \%$ y el $32.9 \%$ de la población objeto de estudio, respectivamente, presentó insatisfacción con su imagen corporal. 
Lo documentado por Durán et al. (23) sobre la insatisfacción con la imagen corporal $(62.8 \%)$ se encuentra mayormente en los estudiantes que se hallan en estado nutricional normal y, sobre éste, el $9 \%$ y el $12 \%$ presentó una sobreestimación de su peso. Esta sobreestimación del tamaño corporal se presenta debido a que los estudiantes de las carreras de la salud, como Nutrición y Dietética, regularmente están controlando su peso corporal y tienen una mayor presión psicosocial para estar en un estado nutricional eutrófico (33).

Por consiguiente, los resultados reflejan que el pertenecer a una carrera que concierne a la salud aumenta la preocupación por asumir y mantener comportamientos saludables, toda vez que el personal sanitario es un modelo no sólo para sus pacientes sino también para la comunidad en general, en cuanto a la promoción y prevención de enfermedades y el logro de un óptimo estado de salud.

Al establecer la relación entre conductas de riesgo y la satisfacción de la imagen corporal, se pudo observar que, a mayor presencia de conductas alimentarias sin riesgo, mayor fue la satisfacción con la imagen corporal; y, de manera contraria, el presentar conductas alimentarias riesgosas aumenta la insatisfacción con la imagen corporal, estableciéndose una relación estadísticamente significativa entre estas dos variables.

De igual manera, Altamirano et al. (34), Moreno y Ortiz (16) y Cano et al. (27), hallaron una relación estadísticamente significativa entre la imagen corporal (IC) y las conductas alimentarias de riesgo (CAR). Sáenz et al. (21) mencionan que, al presentar insatisfacción con la imagen corporal, los individuos evidencian conductas alimentarias de riesgo; hallazgos que fueron asociados principalmente al propósito de bajar de peso e ideales de obtener figuras delgadas. Otro aporte importante brindó León (5), cuya investigación refirió que la insatisfacción con la imagen corporal es un factor para padecer trastornos de la conducta alimentaria y, por tanto, un predictor de las conductas alimentarias de riesgo.

También es importante considerar lo referido por diversos autores que enfatizan en que la mayoría de los jóvenes universitarios se preocupan por la ganancia de peso, la cantidad de calorías que consumen y la carga académica de cada profesión, provocando una percepción errada de la autoimagen y su estado nutricional, lo cual lleva a una insatisfacción con la imagen corporal, siendo esto un factor causal de la aparición de conductas alimentarias de riesgo y trastornos alimenticios $(9,16,17,35)$. Caballero (31) también manifiesta que mayores porcentajes de mujeres con insatisfacción corporal habían realizado conductas alimentarias de riesgo en comparación con quienes estaban satisfechas con sus dimensiones corporales.

El tener una adecuada percepción de la imagen corporal en cuanto al peso, forma y constitución del cuerpo y la despreocupación por obtener una imagen ideal conllevan a la aceptación o conformidad con la propia apariencia física, tal como se evidencio en los resultados de la presente investigación. Esto conduce a que se asuman conductas alimentarias saludables como la adecuada ingesta de alimentos y el ejercicio regular, que mejoraran el estado de salud, impidiendo de esta manera el desarrollo de trastornos alimentarios.

\section{CONCLUSIONES}

En cuanto a las características sociodemográficas de los estudiantes de enfermería, se obtuvo que el $81 \%$ son del género femenino, las edades fluctuaron entre los 16 y los 28 años, el estado civil más frecuente fue el soltero, con el $94 \%$, el estrato socioeconómico más común fue el bajo (1-2), con un $73 \%$, y los estudiantes convivían con sus padres, en el área urbana.

E1 $64 \%$ de los estudiantes presentaron un índice de masa corporal normal y, en menor proporción, se encontraron con sobrepeso (21\%), bajo peso (14 $\%)$ y obesidad tipo 1 (1\%). Estos resultados indican que los estudiantes de enfermería, al mantener una relación de peso y talla adecuada, evitan exponerse a riesgos para la salud que son desencadenados por las alteraciones del estado nutricional, tales como la obesidad, que predisponen riesgos para la aparición de enfermedades crónicas no transmisibles.

En cuanto a las conductas alimentarias de riesgo se puede evidenciar que el $94 \%$ de los participantes presentaron conductas alimentarias sin riesgo, indicando de esta forma que no realizan prácticas extremas para el control de su peso corporal (atracones, dietas restrictivas, ayunos, uso de laxantes, diuréticos,
ISSN 1794-9831

E-ISSN 2322-7028

Vol. 12 No. 2

Jul - Dic 2015

Cúcuta, Colombia 



\section{REFERENCIAS BIBLIOGRÁFICAS}

1. Echegoyen J. Diccionario de psicología científica y filosófica. Explicación de los principales conceptos, tesis y escuelas en el área de la psicología. [Internet]. Bogotá: Universidad Nacional de Educación a Distancia (UNED); 2013 [consultado 13 de octubre de 2014]. Disponible en: http://www.e-torredebabel.com/Psicologia/ Vocabulario/Racionalismo.htm

2. Osorio J, Weisstaub G, Castillo C. Desarrollo de la conducta alimentaria en la infancia y sus alteraciones. Rev. chil. nutr [Internet]. 2002 [consultado 12 de octubre de 2014]; 29 (3): 280-285. Disponible en: http://www.scielo. cl/scielo.php?script=sci_arttext\&pid=S0717-75182002000300002

3. Saucedo T, Unikel C. Conductas alimentarias de riesgo, interiorización del ideal estético de delgadez e índice de masa corporal en estudiantes hidalguenses de preparatoria y licenciatura de una institución privada. Salud Ment [Internet]. 2010 [consultado 18 de septiembre de 2014]; 33 (1): 11-19. Disponible en: http://www.scielo.org.mx/ scielo.php?pid=S0185-33252010000100002\&script=sci_arttext

4. Altamirano M, Vizmanos B, Unikel C. Continuo de conductas alimentarias de riesgo en adolescentes de México. Rev Panam Salud Publica [Internet]. 2011 [consultado 18 de septiembre de 2014]; 30(5): 401-7. Disponible en: http://www.scielosp.org/pdf/rpsp/v30n5/v30n5a01.pdf

5. León Y. Frecuencia de los trastornos de la conducta alimentaria en adolescentes en la Ciudad de Bogotá aplicando la escala de tamizaje: the children`s versión of theeatingattitudes test (cheat). [Tesis]. Bogotá D.C.: Universidad Nacional de Colombia; 2012.

6. López C, Treasure J. Trastornos de la Conducta Alimentaria en Adolescentes. Descripción y manejo. Rev. med. clin. condes [Internet]. 2011 [consultado 18 de septiembre de 2014]; 22(1): 85-97. Disponible en: http://www. clc.cl/Dev_CLC/media/Imagenes/PDF\%20revista\%20m\%C3\%A9dica/2011/1\%20enero/10_PS_Lopez-12.pdf

7. Organización Mundial de la Salud. Prevención de los trastornos mentales Intervenciones efectivas y opciones de políticas. [Internet]. Ginebra: OMS; 2004 [consultado 12 de octubre de 2014]. Disponible en: http://www.who. int/mental_health/evidence/Prevention_of_mental_disorders_spanish_version.pdf

8. Raca F, Silver T. Bulimia nerviosa (Parte 2) Desde la etiología hasta la prevención. Arch. argent. pediatr. [Internet]. 2004 [consultado 12 de octubre de 2014]; 102(6): 468-477. Disponible en: http://www.scielo.org.ar/ scielo.php?script=sci_arttext\&pid $=$ S0325-00752004000600010

9. Domínguez AM. Prevalence of risk of eating disorder in students entering university life. [Tesis Doctoral]. Bogotá DC.: Savannah College; 2012.

10. Quiroga S. Prevalencia e incidencia en la actualidad de los trastornos de la conducta alimentaria. Revista Encrucijadas [Internet]. 2009 [consultado 18 de septiembre de 2014];46. Disponible en: http://www.uba.ar/ encrucijadas/46/sumario/enc46-trastornosalimenticios.php

11. Medina L, Jaimes K. En trastornos de la alimentación, Colombia superaría cifras de países desarrollados. Suvida [Internet]. 2009 [consultado 18 de septiembre de 2014]. Disponible en: http://www.su-vida.com/node/102

12. Sousa VD, Driessnack M, Mendes IAC. Revisión de diseños de investigación. Rev Latino-am Enfermagem [Internet]. 2007 [consultado 18 de septiembre de 2014]; 15(3). Disponible en: http://www.scielo.br/pdf/rlae/ v15n3/es_v15n3a22.pdf

13. Castrillón D, Montaño I, Avendaño G. Validación del bodyshapequestionnaire (cuestionario de la figura corporal) BSQ para la población colombiana. Acta Colombiana de Psicología [Internet]. 2007 [consultado 3 de octubre de 2014]; 10 (1): 15-23. Disponible en: http://portalweb.ucatolica.edu.co/easyWeb2/acta/pdfs/v10n1/ Acta10v1Art2.pdf

14. Hernández L, Londoño C. Imagen corporal, IMC, afrontamiento, depresión y riesgo de TCA en jóvenes universitarios. Anales de Psicología [Internet]. 2013 [consultado 2 de octubre de 2014]; 29 (3): 748-761. Disponible en: http://www.redalyc.org/articulo.oa?id=16728244014

15. Forero M, Rico A, Domínguez A, Baena A. Prevalence of Risk of eating disorder among medical students at the University of La Sabana. [Tesis] Bogotá DC: Savannah College; 2014.

16. Moreno M, Ortiz G .Trastorno alimentario y su relación con la imagen corporal y la autoestima en adolescentes. Ter Psicol [Internet]. 2009 [consultado 2 de octubre de 2014]; 27 (2): 181-190. Disponible en: http://www. scielo.cl/scielo.php?script=sci_arttext\&pid=S0718-48082009000200004\&lng=en\&nrm=iso\&tlng=en

17. Pardo G, Cedeño M. Todo Sobre Técnicas de Investigación. $2^{\mathrm{a}}$ ed. [Tesis]. Caracas DC.: Universidad central de Venezuela; 2014.

18. Corada L, Montedónico A. Study of the contribution of an instrument (Eating Attitudes Test EAT-26, in evaluating changes in adolescents undergoing a program of prevention of obesity. [Tesis]. Santiago DC.: University of Chile; 2007. 
ISSN 1794-9831

E-ISSN 2322-7028

Vol. 12 No. 2

Jul - Dic 2015

Cúcuta, Colombia
19. Veloso V, Lucena $\mathrm{S}$, Santos W. Test de actitudes alimentarias: evidencias de validez de una nueva versión reducida. Interamerican Journal of Psychology [Internet]. 2010 [consultado 3 de octubre de 2014]; 44(1): 28-36. Disponible en: http://www.redalyc.org/pdf/284/28420640004.pdf

20. Galeano D, Krauch C. Actitudes alimentarias y satisfacción con la imagen corporal en mujeres universitarias. Eureka [Internet]. 2010 [consultado 3 de octubre de 2014]; 7(1): 11-38. Disponible en: http://es.scribd.com/ doc/58461306/Eureka-2010\#scribd

21. Sáenz Duran S, González Martínez F, Díaz Cárdenas S. Hábitos y trastornos alimenticios asociados a factores Socio-demográficos, físicos y conductuales en universitarios de Cartagena, Colombia. REV CLÍN MED FAM [Internet]. 2011 [consultado 28 de agosto de 2014]; 4(3): 193-204. Disponible en: http://scielo.isciii.es/pdf/ albacete/v4n3/original2.pdf

22. República de Colombia. Ministerio de Salud. Resolución $N^{\circ} 008430$ de 1993, octubre 4, por la cual se establecen las normas científicas, técnicas y administrativas para la investigación en salud. [Internet]. Santa Fé de Bogotá: El Ministerio; 1993. [consultado 19 de agosto de 2014]. Disponible en: https://www.minsalud.gov. co/Normatividad_Nuevo/RESOLUCION\%208430\%20DE\%201993.pdf

23. Durán S, Bazaez G, Rodríguez M, Figueroa V, Berlanga M, Encina C. Comparación en calidad de vida y estado nutricional entre alumnos de nutrición y dietética y de otras carreras universitarias de la universidad Santo Tomás de Chile. Nutr Hosp. [Internet]. 2012 [consultado 2 de octubre de 2014]; 27 (3): 739-746. Disponible en: http:// scielo.isciii.es/pdf/nh/v27n3/09_original_06.pdf

24. Bernandez M, Miguelez M, Fernández I, Rodríguez G, Carbero J. Evaluación de la distorsión de la imagen corporal en universitarios en relación a sus conocimientos de salud. Nutr. clín. diet. hosp. [Internet]. 2011 [consultado 12 de Octubre de 2014]; 29(2):15-23. Disponible en: http://www.nutricion.org/publicaciones/ revistas/Distorsion_de_la_imagen_corporal.pdf

25. Pérez H, Laguado C, Martínez A. Perfil socioeconómico de los estudiantes de la Universidad Nacional de Colombia. En: Estadísticas e indicadores de la Universidad Nacional de Colombia. $1^{a}$ ed. Bogotá DC.: Universidad Nacional de Colombia; 2010. p. 189-212.

26. Domínguez A. Prevalencia de riesgo de trastornos de la conducta alimentaria en estudiantes que inician la vida universitaria. [Tesis]. Bogotá D.C.: Universidad de la sabana; 2012.

27. Cano AA, Corredor DA, García AM, González M, Lloreda OL, Lucero KV, et al. Factores de Riesgo para trastornos de la alimentación en los alumnos de la Universidad de Manizales. Universidad de Manizales [Internet]. 2006 [consultado 28 de agosto de 2014]. Disponible en: http://ridum.umanizales.edu.co:8080/jspui/ bitstream/6789/1448/1/articulo\%20fr\%20para\%20ta.pdf

28. Franco-Paredes K, Martínez-Moreno AG, Díaz-Reséndiz FJ, López-Espinoza A, Aguilera-Cervantes C, Valdés-Miramontes E. Conductas de riesgo y sintomatología de trastornos del comportamiento alimentario en estudiantes universitarios del Sur de Jalisco, México. Rev. Mex, de trastor. aliment [Internet]. 2010 [consultado 2 de octubre de 2014]; 1(2): 90-101 Disponible en: http://www.scielo.org.mx/scielo.php?script=sci_arttext\&pid $=\mathrm{S} 2007-15232010000200002$

29. Trejo P, Castro D, Facio A, Mollinedo F, Valdez G. Insatisfacción con la imagen corporal asociada al Índice de Masa Corporal en adolescentes. BVSCuba[Internet]. 2010 [consultado 18 de Agosto de 2014]; 26 (3): $144-154$. Disponible en: http://www.bvs.sld.cu/revistas/enf/vol26_3_10/enf07310.htm

30. Fandiño A, Giraldo S, Martínez C, Aux C, Espinosa R. Factores asociados con los trastornos de la conducta alimentaria en estudiantes universitarios en Cali, Colombia. Colomb Med [Internet]. 2007 [consultado 12 de octubre de 2014]; 38 (4) Disponible en: http://bibliotecadigital.univalle.edu.co/bitstream/10893/4249/1/ factores.pdf

31. Caballero L. Relación entre la autopercepción de la imagen corporal y hábitos de alimentación en adolescentes del 5 to año de secundaria de la Institución Educativa Teresa Gonzáles de Fanning. 2007. [Tesis]. Lima D.C.: Universidad Nacional Mayor de San Marcos; 2008.

32. Soto A, Cáceres H, Faure M, Gásquez M, Marengo L. Insatisfacción corporal, búsqueda de la delgadez y malnutrición por exceso, un estudio descriptivo correlacional en una población de estudiantes de 13 a 16 años de la ciudad de Valparaíso. Rev Chil Nutr [Internet]. 2013 [consultado 18 de agosto de 2014]; 40(1):10-15 Disponible en: http:// http://www.scielo.cl/pdf/rchnut/v40n1/art01.pdf

33. Pino J, López M, Moreno A, Faúndez T. Percepción de la imagen corporal, del estado nutricional y de la composición corporal de estudiantes de nutrición y dietética de la Universidad del mar, Talca, Chile. Rev Chil Nutr [Internet]. 2010 [consultado 2 de octubre de 2014]; 37 (3): 1-8. Disponible en: http:// http://www.scielo.cl/ pdf/rchnut/v37n3/art07.pdf 
34. Altamirano M, Vizmanos B, Unikel C. Continuo de conductas alimentarias de riesgo en adolescentes de México. Rev Panam Salud Publica [Internet]. 2011[consultado 18 de septiembre de 2014]; 30(5): 401-7. Disponible en: http://www.scielosp.org/pdf/rpsp/v30n5/v30n5a01.pdf

35. Vaquero R, Alacid F, Muyor J, López P. Imagen corporal; revisión bibliográfica. Nutr Hosp. [Internet]. 2013 [consultado 12 de octubre de 2014]; 28(1):27-35. Disponible en: http://scielo.isciii.es/pdf/nh/v28n1/04revision04. pdf
ISSN 1794-9831

E-ISSN 2322-7028

Vol. 12 No. 2

Jul - Dic 2015

Cúcuta, Colombia 